\title{
Tibor Katriňák
}

Die Kennzeichnung der beschränkten Brouwerschen Verbände

Czechoslovak Mathematical Journal, Vol. 22 (1972), No. 3, 427-434

Persistent URL: http://dml.cz/dmlcz/101112

\section{Terms of use:}

(C) Institute of Mathematics AS CR, 1972

Institute of Mathematics of the Czech Academy of Sciences provides access to digitized documents strictly for personal use. Each copy of any part of this document must contain these Terms of use.

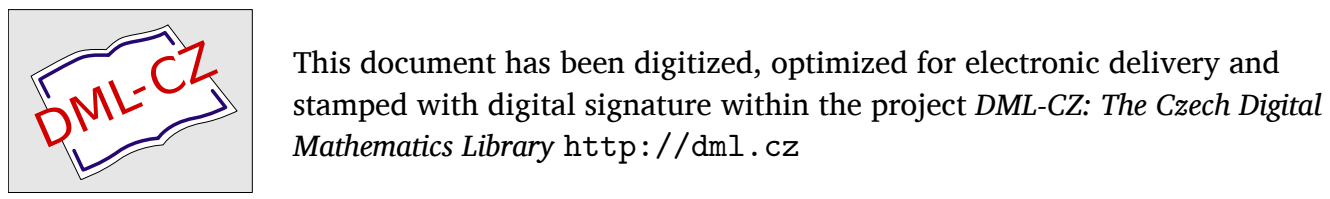




\section{DIE KENNZEICHNUNG \\ DER BESCHRÄNKTEN BROUWERSCHEN VERBÄNDE}

TIBOR KATRIŇÁK, Bratislava

(Eingegangen am 15. Marz 1971)

Alle pseudokomplementären Strukturen - von den distributiven pseudokomplementären Halbverbänden beginnend bis hin zu den beschränkten relativ Stoneschen Verbänden - lassen eine Tripelcharakterisierung im Sinne der Arbeit [3] zu. Die distributiven pseudokomplementären Verbände sind mit Hilfe einer anderen Methode in [4] konstruiert worden. Das Gleiche tun wir hier auch für die beschränkten Brouwerschen Verbände (s. Satz 3). Die beschränkten Brouwerschen Halbverbände waren früher in [5] gekennzeichnet worden. Wie aus einem Beispiel in [5] hervorgeht, braucht ein beschränkter Brouwerscher Halbverband, dessen Filter der dichten Elemente ein Brouwerscher Verband ist, kein Brouwerscher Verband zu sein. Wann dies besteht, besagt uns Satz 2. Alle angeführten Ergebnisse stützen sich auf Satz 1, der diejenigen distributiven pseudokomplementären Verbände beschreibt, die einen Brouwerschen Verband bilden.

\section{VORBEREITENDE BEMERKUNGEN}

Was die Bezeichnung und Terminologie betrifft, werden wir uns am meisten an der Arbeit [3] halten. Wir geben doch einige Begriffe wieder.

Ein Halbverband $\langle L, \cap\rangle$ (ferner nur kurz mit $L$ bezeichnet) heisst distributiv, falls aus $t \geqq x \cap y$ die Existenz von Elementen $x_{1} \geqq x$ und $y_{1} \geqq y$ derart, folgt, dass $t=x_{1} \cap y_{1}$ gilt.

Eine nichtleere Teilmenge $J$ eines Halbverbandes $L$ heisst ein Filter, wenn aus $y \geqq x$ und $x \in J$ stets $y \in J$ folgt, und $x, y \in J$ die Beziehung $x \cap y \in J$ nach sich zieht. Es ist leicht zu sehen, dass für $a \in L$ die Menge $[a)=\{x \in L ; x \geqq a\}$ einen Filter bildet, den wir einen Hauptfilter nennen werden. $F(L)$ wird die Gesamtheit aller Filter von $L$ bezeichnen, die bezüglich der mengentheoretischen Inklusion halbgeordnet ist. Falls der Halbverband $L$ ein grösstes Element 1 enthält, dann bildet $F(L)$ einen Verband, wobei für $J_{1}, J_{2} \in F(L) J_{1} \cap J_{2}$ der mengentheoretische Durch- 
schnitt von $J_{1}$ und $J_{2}$ ist. Setzt man noch die Distributivität von $L$ voraus, dann gilt (s. [2])

$$
J_{1} \cup J_{2}=\left\{t \in L ; t=x \cap y, x \in J_{1} \text { und } y \in J_{2}\right\} .
$$

Wie aus dem folgenden Satz hervorgeht, stellen die distributiven Halbverbände eine Verallgemeinerung von distributiven Verbänden dar.

1.1. (s. [2]). Ein Halbverband List genau dann distributiv, wenn der Filterverband $F(L)$ distributiv ist.

Durch einen Filter $J$ eines distributiven Halbverbandes $L$ kann man eine Kongruenzrelation $\Theta(J)$ in $L$ erklären:

(2) $x \equiv y(\Theta(J))$ genau dann, wenn es ein $v \in J$ gibt, so dass $x \cap v=y \cap v$ gilt. $\Theta(J)$ ist offenbar die kleinste Kongruenzrelation in $L$, die $J$ als Kern enthält.

Ein Filter $J$ eines distributiven Halbverbandes $L$ mit 1 heisst comonomial, wenn jede $\Theta(J)$-Klasse ein grösstes Element besitzt. [a] $\Theta$ wird die Element $a$ enthaltende $\Theta$-Klasse bedeuten.

1.2. Es seien L ein distributiver Halbverband mit 1 und $J_{1}, J_{2}$ zwei Filter von $L$. Es gelte $J_{1} \cup J_{2}=L$ und $J_{1} \cap J_{2}=[d)$ für ein $d \in L$. Dann bildet

$$
\varphi: x \rightarrow[x] \Theta\left(J_{1}\right)
$$

den Teilhalbverband $L_{d}=\left\{x \in J_{2} ; x \leqq d\right\}$ isomorph auf den Faktorhalbverband $L / \Theta\left(J_{1}\right) a b$.

Beweis. Bekanntlich gilt $(x \cap y) \varphi=x \varphi \cap y \varphi$. Angenommen $x \varphi=y \varphi(x, y \in$ $\in L_{d}$ ). Daher ergibt sich nach (2) $x \cap v=y \cap v$ für ein $v \in J_{1}$. Der Distributivität wegen gibt es Elemente $x_{1} \geqq x$ und $v_{1} \geqq v$ mit $y=x_{1} \cap v_{1}$. Folglich ist $x_{1} \equiv$ $\equiv x\left(\Theta\left(J_{1}\right)\right)$, was mit $x=x_{1} \cap w$ für ein $w \in J_{1}$ äquivalent ist. Wegen $x, y \in L_{d}$ ist $w, v_{1} \geqq d$, was $y=x_{1} \cap d=x$ liefert. Also $\varphi$ ist injektiv.

Es sei $[t] \Theta\left(J_{1}\right)$ ein beliebiges Element von $L / \Theta\left(J_{1}\right)$. Es gibt zwei Elemente $t_{1} \in J_{1}$ und $t_{2} \in J_{2}$ mit $t=t_{1} \cap t_{2}$. Wegen $d \equiv 1\left(\Theta\left(J_{1}\right)\right)$ ist $t \equiv t_{2} \cap d\left(\Theta\left(J_{1}\right)\right)$ und $t_{2} \cap d \in$ $\in L_{d}$. Somit ist $\left(t_{2} \cap d\right) \varphi=[t] \Theta\left(J_{1}\right)$ gezeigt, was noch zu beweisen war.

Wir erwähnen noch einige Begriffe. Gibt es für zwei Elemente $a$ und $b$ eines Halbverbandes $L$ ein Element $c=a_{*} b \in L$ mit der Eigenschaft

$$
a \cap x \leqq b \quad \text { genau dann, wenn } x \leqq a_{*} b,
$$

dann ist $a_{*} b$ in $L$ eindeutig bestimmt und heisst ein Relativpseudokomplement. Wenn es für je zwei Elemente $a, b \in L$ das Relativpseudokomplement $a_{*} b$ in $L$ gibt, dann heisst $L$ ein Brouwerscher Halbverband. Ein Halbverband $L$ mit 0 , der für alle $a \in L$ Elemente $a_{*} 0$ besitzt, wird pseudokomplementär genannt. $a_{*} 0$ wird kurz 
mit $a^{*}$ bezeichnet. Brouwersche und pseudokomplementäre Halbverbände besitzen stets 1 .

Einem pseudokomplementären Halbverband $L$ ordnet man zwei wichtige Teilmengen zu: $B(L)=\left\{x \in L ; x=x^{* *}\right\}$ und $D(L)=\left\{x \in L ; x^{*}=0\right\} . B(L)$ bildet bezüglich der induzierten Halbordnung von $L$ eine Boolesche Algebra $\langle B(L), \wedge, \vee$, ', 0,1>, wobei $a \wedge b=a \cap b$ und $a^{*}=a^{\prime}$ für $a, b \in B(L)$ gilt. $D(L)$ stellt einen Filter von $L$ dar, den Filter der dichten Elemente von $L$.

1.3. Für jedes Element $x$ eines distributiven pseudokomplementären Halbverbandes L gibt es ein Element $d \in D(L)$ derart, dass

$$
x=x^{* *} \cap d
$$

gilt.

1.4. Ein Brouwerscher Halbverband ist distributiv.

Beide Sätze findet man in [3, 2.4 und 2.7].

\section{BROUWERSCHE HALBVERBÄNDE MIT 0}

2.1. Seien $J_{1}$ und $J_{2}$ Filter von einem Brouwerschen Halbverband L. Es gelte $J_{1} \cup J_{2}=L$ und $J_{1} \cap J_{2}=[d)$ für ein $d \in L$. Dann sind die beiden Filter $J_{1}$ und $J_{2}$ comonomial. Ferner ist $d_{*} t$ für $t \in J_{2}$ das grösste Element in der Klasse $[t] \Theta\left(J_{1}\right)$.

Beweis. Es genügt zu zeigen, dass z. B. $J_{1}$ ein comonomialer Filter ist. Sei $p \in L$. Wegen $J_{1} \cup J_{2}=L$ gibt es zwei Elemente $q \in J_{1}$ und $t \in J_{2}$ derart, dass $p=$ $=q \cap t$ gilt. Daher ergibt sich $p \equiv t\left(\Theta\left(J_{1}\right)\right) . d \in J_{1}$ und $d \cap t=d \cap\left(d_{*} t\right)$ impliziert $t \equiv d_{*} t\left(\Theta\left(J_{1}\right)\right)$. Angenommen, dass $r \equiv p\left(\Theta\left(J_{1}\right)\right)$ für ein $r \in L$ gilt. Folglich ist $r \equiv d_{*} t\left(\Theta\left(J_{1}\right)\right)$. Demnach gibt es ein $w \in J_{1}$ mit

$$
r \cap w=\left(d_{*} t\right) \cap w .
$$

Der Distributivität von $L$ wegen gibt es wiederum Elemente $v \geqq w$ und $r_{1} \geqq r$ mit

$$
d_{*} t=r_{1} \cap v \text {. }
$$

Daraus folgt $r_{1} \geqq d_{*} t$ und $r_{1} \equiv d_{*} t\left(\Theta\left(J_{1}\right)\right)$. Ferner $t \leqq d_{*} t \leqq v, t \in J_{2}$ und $w \leqq v$ liefert uns $v \geqq d$. Weiterhin gilt

$$
d \cap r_{1}=d \cap\left(v \cap r_{1}\right)=d \cap d_{*} t \leqq t .
$$

Daher ergibt sich $r \leqq r_{1} \leqq d_{*} t$. Damit haben wir bewiesen, dass $d_{*} t$ das grösste Element in der Klasse $[p] \Theta\left(J_{1}\right)=[t] \Theta\left(J_{1}\right)$ ist. 
Es sei $L$ ein distributiver pseudokomplementärer Halbverband. Bezeichne $a \Psi^{L}=$ $=\{x \in D(L) ; x \geqq a\}$ für $a \in B(L)$. Es ist klar, dass $a \Psi^{L}$ ein Filter von $D(L)$ mit

ist (s. [3]).

$$
a \Psi^{L} \cup a^{*} \Psi^{L}=0 \Psi^{L}=D(L)
$$

2.2. Es sei L ein distributiver pseudokomplementärer Halbverband, wobei der Filter $D(L)$ der dichten Elemente von L einen Brouwerschen Halbverband bildet. Es sei noch $a \Psi^{L} \cap a^{*} \Psi^{L}$ für jedes $a \in B(L)$ ein Hauptfilter in $D(L)$. Dann stellt $L$ einen Brouwerschen Halbverband (mit 0) dar.

Bewe is. Wegen der Voraussetzung und $a \Psi^{L} \cup a^{*} \Psi^{L}=D(L)(a \in B(L))$ bildet $a \Psi^{L}$ nach 2.1 einen comonomialen Filter in $D(L)$ für alle $a \in B(L)$. Unsere Behauptung folgt nun aus [3, 4.2 und Satz 5.9].

Satz 1. Es sei L ein distributiver pseudokomplementärer Verband. L ist genau dann brouwersch, wenn $D(L)$ ein Brouwerscher Verband ist.

Beweis folgt aus 2.2. Wir bringen noch einen anderen, konstruktiven Beweis. Es ist zu zeigen, dass für alle $x, y \in L \quad x_{*} y$ in $L$ existiert.

Fall 1. $x \in L, y=a \in B(L)$. Wir zeigen, dass $x_{*} a \in B(L)$ und

$$
x_{*} a=x^{*} \vee a=\left(x^{*} \cup a\right)^{* *}
$$

gilt. Bekanntlich ist $x^{* *} \geqq x$ und $x^{* *} \in B(L)$. Demzufolge gilt

$$
x \cap\left(x^{*} \vee a\right) \leqq x^{* *} \cap\left(x^{*} \vee a\right)=\left(x^{* *} \cap x^{*}\right) \vee\left(x^{* *} \cap a\right) \leqq a .
$$

Umgekehrt, sei $x \cap z \leqq a$ für ein $z \in L$. Wegen

$$
(x \cap z) \leqq(x \cap z)^{* *}=x^{* *} \cap z^{* *} \leqq a^{* *}=a
$$

(s. [3, 2.3]) gilt dann $z \leqq z^{* *} \leqq x^{*} \vee a$, weil $x^{* *}, z^{* *}$ und $a$ in der Booleschen Algebra $B(L)$ liegen. Damit haben wir (2.1) nachgewiesen.

Fall 2. $x=a$ und $a \in B(L), y=d$ und $d \in D(L) . x_{*} y$ existiert, liegt in $D(L)$ und gilt

$$
a_{*} d=\left(a \cup a^{*}\right)_{*}\left(a^{*} \cup d\right) .
$$

Wegen $a \cup a^{*}, a^{*} \cup d \in D(L)$ und der Annahme nach existiert das Element $\left(a \cup a^{*}\right)_{*}\left(a^{*} \cup d\right)$ in $D(L)$. Offensichtlich

$$
\begin{gathered}
a \cap\left[\left(a \cup a^{*}\right)_{*}\left(a^{*} \cup d\right)\right]=a \cap\left(a \cup a^{*}\right) \cap\left[\left(a \cup a^{*}\right)_{*}\left(a^{*} \cup d\right)\right] \leqq \\
\leqq a \cap\left(a^{*} \cup d\right)=a \cap d \leqq d .
\end{gathered}
$$

Es sei für $t \in D(L) a \cap t \leqq d$. Demzufolge

$$
(a \cap t) \cup a^{*}=\left(a \cup a^{*}\right) \cap\left(t \cup a^{*}\right) \leqq a^{*} \cup d .
$$


Daraus ergibt sich $t \leqq a^{*} \cup t \leqq\left(a \cup a^{*}\right)_{*}\left(a^{*} \cup d\right)$. Falls $a \cap z \leqq d$ für ein $z \in L$ ist, dann gilt für $t:=z \cup\left(a \cup a^{*}\right)_{*}\left(a^{*} \cup d\right) \in D(L)$ auch $a \cap t \leqq d$, woraus schon $z \leqq t \leqq\left(a \cup a^{*}\right)_{*}\left(a^{*} \cup d\right)$ folgt. Damit ist (2.2) bewiesen.

Angenommen, dass die Elemente $x_{*} y$ und $x_{*} z$ in $L$ existieren $(x, y, z \in L)$. Wir zeigen, dass auch das Element $x_{*}(y \cap z)$ in L existiert und gilt

$$
x_{*}(y \cap z)=\left(x_{*} y\right) \cap\left(x_{*} z\right) .
$$

Offensichtlich $x \cap\left(x_{*} y\right) \cap\left(x_{*} z\right) \leqq y \cap z . x \cap t \leqq y \cap z(t \in L)$ impliziert $x \cap t \leqq y$ und $x \cap t \leqq z$, woraus schon $t \leqq\left(x_{*} y\right) \cap\left(x_{*} z\right)$ folgt. (2.3) ist nachgewiesen.

Schliesslich nehmen wir an, dass das Element $x_{*}\left(y_{*} z\right)$ in Lexistiert. Dann existiert in $L$ auch $(x \cap y)_{*} z$ und gilt

$$
(x \cap y)_{*} z=x_{*}\left(y_{*} z\right) .
$$

Offenbar $(x \cap y) \cap\left[x_{*}\left(y_{*} z\right)\right]=y \cap\left[x \cap x_{*}\left(y_{*} z\right)\right] \leqq y \cap\left(y_{*} z\right) \leqq z$. Sei für $t \in L$ $(x \cap y) \cap t \leqq z$. Daraus ergibt sich $x \cap t \leqq y_{*} z$ und folglich auch $t \leqq x_{*}\left(y_{*} z\right)$. (2.4) ist bewiesen.

Nun können wir die bisherigen Resultate zusammenfassen. Seien $x, y \in L$. Es gibt nach 1.3 Elemente $d_{1}, d_{2} \in D(L)$ derart, dass $x=x^{* *} \cap d_{1}$ und $y=y^{* *} \cap d_{2}$ gelten wird. Wegen (2.2) existiert das Element $x_{*} d_{2}$ in $L$ und gilt $x_{*}^{* *}\left(d_{1 *} d_{2}\right)=$ $=\left(x^{* *} \cap d_{1}\right)_{*} d_{2}=x_{*} d_{2}$ (s. (2.4)). Das Element $x_{*} y^{* *}$ existiert auch in $L(\mathrm{~s} .(2.1))$. Mithin existiert $x_{*} y$ in $L($ s. (2.3)) und gilt

$$
\begin{aligned}
x_{*} y & =\left(x_{*} y^{* *}\right) \cap\left(x_{*} d_{2}\right)=\left(x^{*} \vee y^{* *}\right) \cap x^{* *}{ }_{*}\left(d_{1 *} d_{2}\right)= \\
& =\left(x^{*} \vee y^{* *}\right) \cap\left[\left(x^{* *} \cup x^{*}\right)_{*}\left(x^{*} \cup\left(d_{1 *} d_{2}\right)\right)\right] .
\end{aligned}
$$

In [5] wurde anhand eines Beispieles gezeigt, dass es Brouwersche Halbverbände $L$ mit 0 und mit einem Brouwerschen Verband $D(L)$ gibt, die keinesfalls Verbände zu sein brauchen. Dazu sagt uns der folgende Satz

Satz 2. Es sei L ein Brouwerscher Halbverband mit 0, wobei der Filter der dichten Elemente $D(L)$ einen Verband bildet. List genau dann ein Brouwerscher Verband, wenn für alle $a \in B(L)$ a $\Psi^{L} \cap a^{*} \Psi^{L}$ einen Hauptfilter von $D(L)$ darstellt.

Beweis. Die notwendige Bedingung folgt aus $\left[a \cup a^{*}\right)=a \Psi^{L} \cap a^{*} \Psi^{L}$. Die hinreichende Bedingung ergibt sich aus [3, Satz 5.12] und Satz 1.

\section{TRIPELCHARAKTERISIERUNG DER BROUWERSCHEN VERBÄNDE MIT 0}

Die Teilmengen $B(L), D(L)$ und die Abbildung $\Psi^{L}$ von $B(L)$ in den Filterverband $F(D(L))$ ist von der grundlegenden Bedeutung für die distributiven pseudokomplementären und Brouwerschen (Halb-) Verbände, wie aus [3], [4] und [5] zu ent- 
nehmen ist. Die erste Kennzeichnung der Brouwerschen Halbverbände mit 0 in dieser Richtung ist in [5] unternommen worden. In [3] ist dies ganz allgemein für verschiedene Strukturen besprochen. In [4] ist eine andere Methode zur Konstruierung der distributiven pseudokomplementären Verbände entwickelt worden. Wir möchten hier noch in dieser Richtung einen Beitrag für die Brouwerschen Verbände mit 0 leisten. Zuerst einige Begriffe und Resultate aus [4].

Seien $B=\left\langle B, \vee, \wedge,{ }^{\prime}, 0,1\right\rangle$ ein Boolescher Verband, $D=\left\langle D, \cup, \cap,{ }_{*}, 1\right\rangle$ ein Brouwerscher Verband und $\Phi$ ein $(e, \cup)$-Homomorphismus von $B$ in $F(D)$ (d. h. $0 \Phi=[1), 1 \Phi=D,(x \vee y) \Phi=x \Phi \cup y \Phi)$. Ein Tripel $\langle B, D, \Phi\rangle$ wird ein $b$-Tripel heissen, wenn für alle $a \in B a \Phi \cap a^{\prime} \Phi$ ein Hauptfilter ist. Es ist leicht zu sehen, dass im Falle eines Brouwerschen Verbandes $L$ mit $0\left\langle B(L), D(L), \Phi^{L}\right\rangle$ für $a \Phi^{L}:=a^{*} \Psi^{L}$ $(a \in B(L))$ ein b-Tripel bildet.

Für ein b-Tripel $\langle B, D, \Phi\rangle$ und $a \in B$ bezeichne $d_{a} \in D$ das Element, das durch

$$
\left[d_{a}\right)=a \Phi \cap a^{\prime} \Phi
$$

bestimmt ist. Ferner bildet für ein $d \in D$ und $a \in B$

$$
[d) \cap a \Phi
$$

einen Hauptfilter von $D$. Es bezeichne $d \varrho_{a}$ das erzeugende Element dieses Hauptfilters. (Es gilt nämlich $\left[d_{a} \cup d\right)=([d) \cap a \Phi) \cap\left([d) \cap a^{\prime} \Phi\right)$ und $([d) \cap a \Phi) \cup([d) \cap$ $\left.\cap a^{\prime} \Phi\right)=[d)$. Beide Filter sind dann Hauptfilter (s. [3, 1.9]).) Ferner bezeichne noch $D_{a}=\left\{x \in a \Phi ; x \leqq d_{a}\right\}(a \in B)$.

Satz 3. Es sei $\langle B, D, \Phi\rangle$ ein b-Tripel. Bezeichne L die Gesamtheit aller Paare $\langle a, d\rangle$ mit $a \in B$ und $d \in D_{a}$. Dann ist die Menge L durch

$$
\langle a, d\rangle \leqq\langle b, e\rangle \text { genau dann, wenn } a \leqq b \text { und } d \leqq e \varrho_{a}
$$

halbgeordnet. Bezüglich dieser Halbordnung bildet L einen Brouwerschen Verband mit 0 , wobei für $x=\langle a, d\rangle, y=\langle b, e\rangle \in L$

$$
\begin{aligned}
& x \cap y=\left\langle a \wedge b,(d \cap e) \varrho_{a \wedge b}\right\rangle, \\
& x \cup y=\left\langle a \vee b,\left(d \varrho_{b^{\prime}} \cap e\right) \cup\left(e \varrho_{a^{\prime}} \cap d\right)\right\rangle, \\
& x^{*} \quad=\left\langle a^{\prime}, d_{a^{\prime}}\right\rangle, \\
& \langle 0,1\rangle \leqq x \leqq\langle 1,1\rangle
\end{aligned}
$$

und

$$
x_{*} y=\left\langle a^{\prime} \vee b, d_{*}\left(e \varrho_{a}\right) \cap d_{a^{\prime} \vee b}\right\rangle
$$

in L gilt. 
Beweis. Nach $[4,4.3]$ bildet die Menge Leinen distributiven pseudokomplementären Verband, in dem die Beziehungen (3.1)-(3.5) gelten. Ferner gilt nach [4, Satz 2]

$$
\begin{aligned}
& B(L)=\left\{\langle a, d\rangle \in L ; d=d_{a}, a \in B\right\}, \\
& D(L)=\{\langle 1, d\rangle \in L ; d \in D\} \text { und }\left\langle a, d_{a}\right\rangle \Phi^{L}=\{\langle 1, d\rangle \in L ; d \in a \Phi\} .
\end{aligned}
$$

Daher folgt $B(L) \simeq B$ und $D(L) \simeq D$. Also $D(L)$ bildet sogar einen Brouwerschen Verband. Nach Satz 1 ist $L$ ein Brouwerscher Verband mit 1 . Es bleibt noch zu zeigen, dass die Operation des Relativpseudokomplementes in $L$ durch (3.6) gegeben ist. Zuerst betrachten wir zwei Spezialfälle:

Fall 1. $x=\langle a, d\rangle \in L, y=\langle 1, e\rangle \in D(L)$. Es ist $x_{*} y=\left\langle 1, d_{*}\left(e \varrho_{a}\right)\right\rangle$ zu zeigen. Wegen (3.1) und (3.2) gilt

$$
\langle a, d\rangle \cap\left\langle 1, d_{*}\left(e \varrho_{a}\right)\right\rangle=\left\langle a,\left(d \cap e \varrho_{a}\right) \varrho_{a}\right\rangle=\left\langle a, d \cap e \varrho_{a}\right\rangle \leqq\langle 1, e\rangle .
$$

Angenommen, dass $\langle a, d\rangle \cap\langle c, f\rangle \leqq\langle 1, e\rangle$ für ein $\langle c, f\rangle \in L$ ist. Wegen der Distributivität von $L$ gilt

$$
\begin{array}{r}
\langle a, d\rangle \cap(\langle c, f\rangle \cup\langle 1, e\rangle)=\langle a, d\rangle \cap\left\langle 1, e \cup\left(e \varrho_{c^{\prime}} \cap f\right)\right\rangle= \\
=\left\langle a,(d \cap e) \varrho_{a} \cup\left(d \cap f \cap e \varrho_{c^{\prime}}\right) \varrho_{a}\right\rangle \leqq\langle 1, e\rangle .
\end{array}
$$

Die letzte Beziehung ist nach (3.1) mit

$$
d \cap f \varrho_{a} \cap e \varrho_{c} \varrho_{a} \leqq e \varrho_{a}
$$

und dies schliesslich mit

$$
f \varrho_{a} \cap e \varrho_{c^{\prime}} \varrho_{a} \leqq d_{*}\left(e \varrho_{a}\right)
$$

äquivalent. Setzen wir nun $t_{1}:=f \varrho_{a} \cup\left(d_{*}\left(e \varrho_{a}\right)\right)$ und $t_{2}:=e \varrho_{a} \varrho_{c}, \cup\left(d_{*}\left(e \varrho_{a}\right)\right)$. Daher ergibt sich $t_{1} \cap t_{2}=d_{*}\left(e \varrho_{a}\right)$. Setzt man ferner $p_{1}:=\left(d_{*}\left(e \varrho_{a}\right)\right) \varrho_{c}$ und $p_{2}:=$ $:=\left(d_{*}\left(e \varrho_{a}\right)\right) \varrho_{c^{\prime}}$, dann gilt

$$
p_{1} \cap p_{2}=d_{*}\left(e \varrho_{a}\right) \text {. }
$$

Wegen $f \varrho_{a} \in c \Phi$ ist $p_{1} \leqq t_{1}$. Analog wegen $e \varrho_{a} \varrho_{c^{\prime}} \in c^{\prime} \Phi$ gilt $p_{2} \leqq t_{2} . e \varrho_{a} \leqq d_{*}\left(e \varrho_{a}\right)$ impliziert $e \varrho_{a} \varrho_{c^{\prime}} \leqq\left(d_{*}\left(e \varrho_{a}\right)\right) \varrho_{c^{\prime}}$, woraus schon $p_{2}=t_{2}$ folgt. Offenbar ist $p_{2} \in c^{\prime} \Phi$ und $p_{1} \in c \Phi$. Ferner gilt $p_{1} \equiv t_{1}\left(\Theta\left(c^{\prime} \Phi\right)\right)$. Nach 2.1 ist $d_{c *} p_{1}$ das grösste Element in der Klasse $\left.\left[p_{1}\right] \Theta\left(c^{\prime} \Phi\right)\right)$. Daher ergibt sich

Folglich ist

$$
t_{1} \leqq d_{c *} p_{1}
$$

$$
f \leqq d_{c *} p_{1}
$$

Wegen $f \leqq d_{c}$ gilt

$$
f \leqq d_{c} \cap p_{1} \leqq p_{1}=\left(d_{*}\left(e \varrho_{a}\right)\right) \varrho_{c}
$$


Die letzte Beziehung mit (3.1) liefert uns

$$
\langle c, f\rangle \leqq\left\langle 1, d_{*}\left(e \varrho_{a}\right)\right\rangle .
$$

Fall 2. $x=\langle a, d\rangle \in L, y=\left\langle b, d_{b}\right\rangle \in B(L)$. $L$ stellt einen Brouwerschen Verband mit 0 dar. Im Beweis von Satz 1 (s. (2.1)) haben wir gezeigt

$$
x_{*} y=\left(x^{*} \cup y\right)^{* *} .
$$

Wegen (3.3) und (3.4) erhält man

$$
x_{*} y=\left(\left\langle a^{\prime}, d_{a^{\prime}}\right\rangle \cup\left\langle b, d_{b}\right\rangle\right)^{* *}=\left\langle a^{\prime} \vee b, d_{a^{\prime} \vee b}\right\rangle .
$$

Betrachten wir nun ganz allgemein $x=\langle a, d\rangle$ und $y=\langle b, e\rangle$ aus $L$. Offenbar gilt $y=\left\langle b, d_{b}\right\rangle \cap\langle 1, e\rangle$. Daher erhält man (s. (2.3))

$$
\begin{gathered}
x_{*} y=\langle a, d\rangle_{*}\left(\left\langle b, d_{b}\right\rangle \cap\langle 1, e\rangle\right)=\langle a, d\rangle_{*}\left\langle b, d_{b}\right\rangle \cap \\
\cap\langle a, d\rangle_{*}\langle 1, e\rangle=\left\langle a^{\prime} \vee b, a_{a^{\prime} \vee b}\right\rangle \cap\left\langle 1, d_{*}\left(e \varrho_{a}\right)\right\rangle= \\
=\left\langle a^{\prime} \vee b,\left(d_{*}\left(e \varrho_{a}\right) \cap d_{a^{\prime} \vee b}\right) \varrho_{a^{\prime} \vee b}\right\rangle=\left\langle a^{\prime} \vee b, d_{*}\left(e \varrho_{a}\right) \cap d_{a^{\prime} \vee b}\right\rangle,
\end{gathered}
$$

weil $e \leqq d_{*}\left(e \varrho_{a}\right)$ und $e \in b \Phi \subseteq\left(a^{\prime} \vee b\right) \Phi$ gilt.

\section{Literatur}

[1] G. Birkhoff, Lattice theory, Amer. Math. Soc. Colloquium Publications, vol. 25, third edition 1967.

[2] T. Katriňák, Pseudokomplementäre Halbverbände, Mat. časop. 18 (1968), 121-143.

[3] T. Katriňák, Die Kennzeichnung der distributiven pseudokomplementären Halbverbände, J. reine und angew. Math. 241 (1970), 160-179.

[4] T. Katriňák, Über eine Konstruktion der distributiven pseudokomplementären Verbände, Math. Nachr. 53 (1972), 85-99.

[5] W. C. Nemitz, Implicative semi-lattices, Trans. Amer. Math. Soc. 117 (1965), 128-142.

Anschrift des Verfassers: Bratislava-Mlynská dolina, Matematický pavilon, ČSSR, (Prírodovedecka fakulta UK). 